\title{
Acute Myeloid Leukaemia complicated by myeloid sarcoma presenting as acute flaccid paralysis
}

\author{
Ediriweera HMA ${ }^{1}$, Mohideen MS ${ }^{1}$, Fernando HSST ${ }^{2}$, Pathirana KD ${ }^{1}$, Weerarathna TP \\ ${ }^{1}$ University Medical Unit, ${ }^{2}$ Haematology Unit, Teaching Hospital Karapitiya, Galle, Sri Lanka. \\ Correspondence: $\quad$ Dr.HMA Ediriweera \\ e-mail: anushkaediriweera@gmail.com
}

\section{Introduction}

Myeloid sarcoma (MS) is a rare soft tissue tumour. Its presentation can vary from an isolated extra medullary leukaemic tumour, concurrently with or at relapse of acute myeloid leukaemia (AML) (1). The clinical presentation of MS varies according to the site of involvement. Common sites include sub-periosteum of skull, paranasal sinuses, sternum, ribs, vertebrae and pelvis. Lymph nodes and skin are also common sites. We report a case of MS in a sixteen year old girl presenting with acute flaccid paraparesis due to cord compression.

\section{Case report}

A 16-year-old, previously healthy girl presented with acute weakness and numbness of lower limbs and lower trunk for few hours. She had not passed urine for 12 hours. She had history of varicella infection one week prior but not sought any medical advice. Two days before admission, she was treated for dyspepsia and epigastric pain by her general practitioner. She denied having any trauma, history of exertional dyspnoea, recurrent infections other than the varicella infection or any abnormal bleeding manifestations.

On examination, she was febrile and anicteric. Healed ulcers of varicella infection were evident. There were flaccid hyporeflexic lower limbs with sensory loss of all modalities up to T6. Rest of the neurological examination including the upper limbs was normal. There was no lymphadenopathy or hepatosplenomegaly. Bladder was palpable extending up to umbilicus. Cardiovascular and respiratory system examination was normal.
Preliminary investigations revealed pancytopaenia $\left(\mathrm{Hb}-7 \mathrm{~g} / \mathrm{dL}, \mathrm{WBC}-6.1 \times 10^{9} / \mathrm{L}\right.$, platelet $\left.-57 \times 10^{9} / \mathrm{L}\right)$ and her peripheral blood film showed normal red cell morphology, leukopaenia and neutropaenia with some blast cells and moderately low platelets. The haematological findings suggested acute leukaemia. Bone marrow aspiration and trephine biopsy confirmed acute myeloid leukaemia with maturation (FAB AML M2). Chest radiograph showed retro cardiac shadow separated from cardiac border (Figure 1). An urgent Magnetic Resonance Imaging (MRI) of the spine showed paraspinal mass extending from $\mathrm{T} 2$ to $\mathrm{T} 7$. The mass extended into the spinal canal through left T4/T5 and T5/T6 exit foramina and there was an evidence of severe cord compression at the same level. Biopsy of the paraspinal mass under the ultrasound guidance was done and the histology was compatible with myeloid sarcoma.

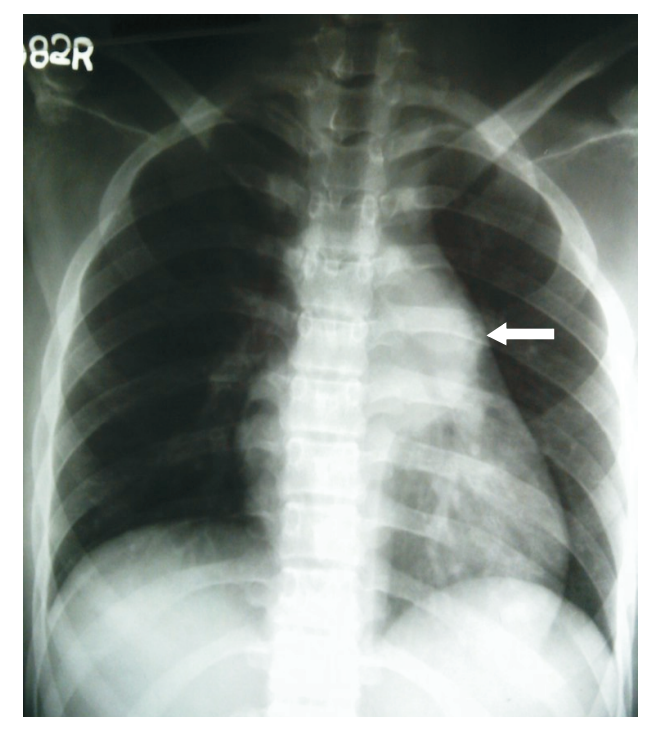

Figure 1: Retrocardiac shadow (arrow) 
The diagnosis of myeloid sarcoma concurrent with acute myeloid leukaemia was made based on above investigations and the patient was referred to neurosurgeon and oncologist for urgent de-bulking surgery and radiotherapy. Patient underwent laminectomy followed by radiotherapy.

\section{Discussion}

Myeloid sarcoma is defined as an extramedullary tumour consisting of myeloid blast cells with or without maturation, with associated effacement of tissue architecture (2). MS may be secondary to AML, chronic myeloid leukaemia (CML), blast crisis or myelodysplastic syndrome in the descending order of frequency (3). The other sites of occurrence of MS include mediastinum, pancreas, parotid gland and uterus (4). The presentation of myeloid sarcoma as acute paralysis due to cord compression by MS is rare and diagnostically challenging (5).

As this patient had varicella infection one week prior to the presentation with acute flaccid paralysis, the first differential diagnosis was post-viral transverse myelitis. But pancytopenia and blast cells in blood picture suggested an alternative diagnosis. Imaging revealed a paraspinal mass causing cord compression and the possibility of ganglioneuroblastoma or ganglioneuroma were considered. With the haematological and blood picture findings suggestive of AML, it was necessary to differentiate between ganglioneuroblastoma infiltrating marrow causing pancytopenia and leukemic deposits causing cord compression. Findings of the biopsy of the paraspinal mass and bone marrow aspiration finally confirmed the later diagnosis.

Finding of myeloid cells with different maturation with strong positivity of myeloperoxidase staining of paraspinal mass biopsy (Figure 2) further supported the diagnosis of MS. The varicella infection prior to this presentation could be due to immunosuppression secondary to leukopenia with AML.

The diagnosis of AML with maturation (classified according to the WHO classification) was made with the morphological appearance of blasts of more than $20 \%$ in the bone marrow with more than $10 \%$ maturing granulocyte component and less than $20 \%$ of monocyte lineage.
Patient underwent debulking surgery followed by radiotherapy under the oncologist's supervision. But the observed clinical improvement from paralysis was minimal two weeks following therapy.

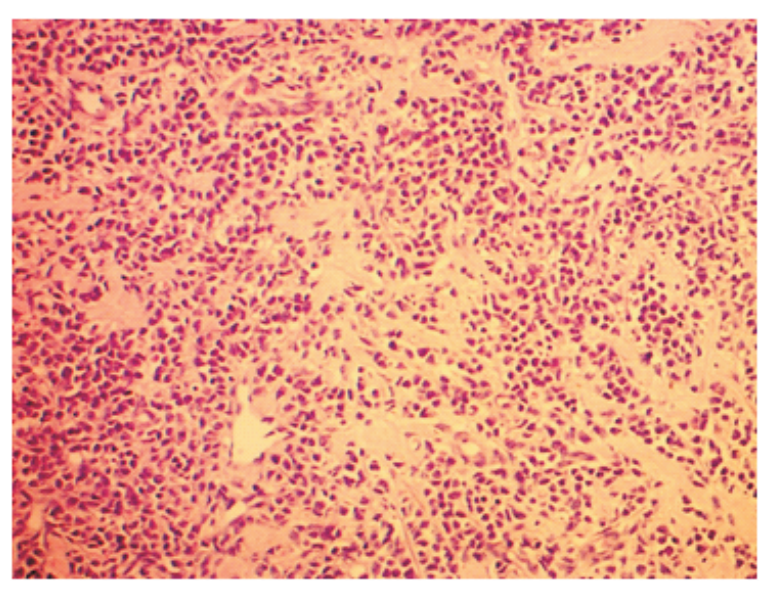

Figure 2(a): Haemotoxyline and Eosin stain

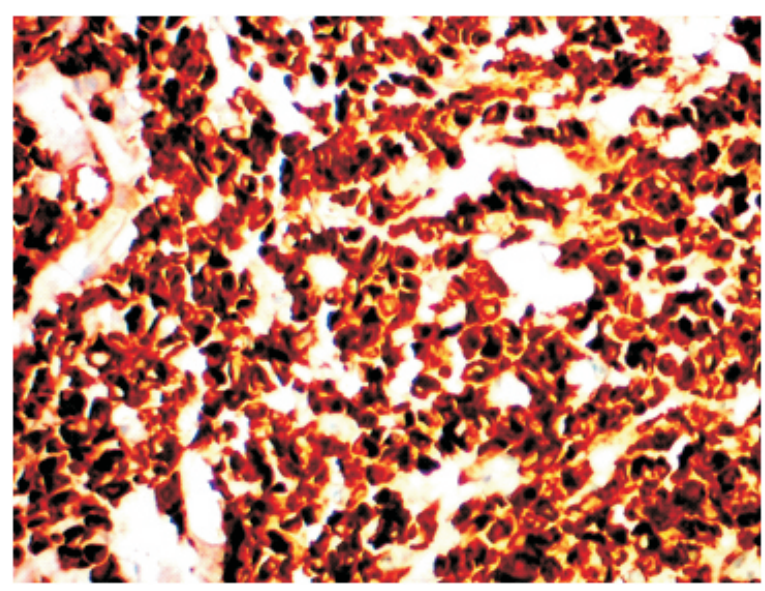

Figure 2(b): Myeloperoxidase staining

Figure 2: Biopsy of paraspinal mass.

\section{Conclusion}

The acute flaccid paralysis following viral infection is not always transverse myelitis. The high degree of suspicion to exclude local causes is important to prevent / reduce rate of permanent neurological damage. Early diagnosis and intervention will change the prognosis from "poor" to reasonable outcome even though the diagnosis is challenging. 


\section{References}

1. Ansari-Lari MA, Yang CF, Tinawi-Aljundi R, Cooper L, Long P, Allan RH, et al. FLT3 mutations in myeloid sarcoma. British Journal of Haematology, 2004; 126: 785-91.

2. Pileri SA, Orazi A, Falini B. Myeloid sarcoma. WHO classification of Tumours of Haematopoietic and Lymphoid Tissues. Lyon: IARC Press; 2008: 140-1.

3. Singhal RL, Monaco SE, Pantanowitz L, Cytopathology of Myeloid Sarcoma: A study of 16 cases, Journal of the American Society of Cytopathology, (2014), doi: 10.1016/j.jasc.2014.10.001.
4. Massimo Breccia, Franco Mandelli, Maria C. Petti, MariellaD' Andrea, Edoardo Pescarmona, Stefano A. Pileri, Ida Carmosino, Eleonora Russo, Paolo D Fabritiis, Giuliana Alimena. Clinicopathological characteristics of myeloid sarcoma at diagnosis and during follow-up: report of 12 cases from a single institution. Leukaemia Research, 2004; 28: 1165-9.

5. Landis DM, Aboulafia DM. Granulocytic sarcoma: An unusual complication of a leukaemic myeloid leukaemia causing spinal cord compression. A case report and literature review. Leukaemic Lymphoma, Oct 2003;44(10): 1753-60. 